\title{
Pelo telefone - histórias e relatos sobre o uso de celulares como ferramenta de ensino e aprendizagem de língua inglesa no Brasil
}

\author{
Maria do Carmo Ferreira Xavier ${ }^{1}$, Lucimeri Ricas Dias ${ }^{2}$ \\ 1Pós-Graduação em Tecnologias da Informação Aplicadas à Educação (PGTIAE-iNCE) \\ ${ }^{2}$ Pós-Graduação em História das Ciências e das Técnicas e Epistemologia (HCTE) \\ Universidade Federal do Rio de Janeiro (UFRJ) \\ docarmoferreira@gmail.com, luricas@gmail.com
}

\begin{abstract}
This paper describes experiences with the mobile phone as a tool for teaching English between 2009 and 2012. Those experiences were conducted with adolescents aged between 12 and 14 years old, in a language school in Rio de Janeiro. In this description the following items will be encompassed: the activities created, the planning and implementation stages and the difficulties throughout the implementation. Also, the principles of Mobile Assisted Language Learning (MALL) will be described, as well as possibilities for integration of information technologies to the classroom. Finally, this paper will describe the pedagogical gains and other possibilities integrating mobile devices to the classroom.
\end{abstract}

Resumo. Este artigo descreve experiências com o telefone celular como ferramenta de ensino de língua inglesa entre 2009 e 2012. Estas experiências foram realizadas com adolescentes de 12 a 14 anos de idade, em uma escola de idiomas no Rio de Janeiro. Neste relato serão descritas: as atividades criadas, o processo de planejamento e implementação e as dificuldades ao longo da implementação. Também serão descritos os princípios norteadores do Mobile Assisted Language Learning (MALL), bem como outras possibilidades integração de tecnologias da informação à sala de aula.

\section{Introdução}

Segundo a Anatel [2011], no final de 2011, o Brasil contava com 242,23 milhões de acessos do Serviço Móvel Pessoal (SMP), com crescimento de 16,2\% em relação ao ano anterior. Em 2010, este crescimento foi semelhante: 16,7\% em relação a 2009. Essa evolução teve forte contribuição da disponibilidade cada vez maior de redes $3 \mathrm{G}$ no país, que ampliaram o acesso à banda larga móvel. Também, segundo a Agência Nacional de Telecomunicações, 59.235 escolas públicas urbanas possuíam conexão à internet banda larga em dezembro de 2011. 
O celular se popularizou, e foi, aos poucos, incorporado à cultura brasileira: a mobilidade deixou de ser apenas uma característica dos novos aparelhos eletrônicos. Ela é também um valor. O celular (ou telemóvel, como é chamado em muitos países de língua portuguesa) inspirou outras invenções que trazem a mesma filosofia: tablets, games, netbooks etc. Todos são portáteis. Além disso, a convergência também é outro valor apresentado ao mundo pelos celulares: não basta fazer e receber ligações. Um bom celular precisa ser capaz de tirar fotos de qualidade, fazer cálculos matemáticos, armazenar documentos importantes e reproduzir arquivos de músicas.

Por causa do telemóvel novos hábitos foram incorporados ao dia-a-dia das pessoas: quem já não se deparou com estudantes fotografando as anotações de um professor em seu quadro, ao invés de copiá-las? E o que dizer das inúmeras campanhas sobre educação no trânsito, que incluem, hoje, novas normas com relação ao uso de celulares ao volante? Também não podemos deixar de notar as inúmeras regras de etiqueta criadas a partir do celular: atendê-lo enquanto se assiste a palestras em escolas ou igrejas, por exemplo, é considerado um mau hábito. Prestar atenção à conversa de alguém ao celular também pode ser muito grosseiro. Ainda há aqueles que, para desagrado de muitos, insistem em usar a internet do celular ou trocar mensagens de texto, mesmo quando estão cercados de amigos e familiares. Também não nos esqueçamos de mencionar as cenas comuns de pessoas que, ao preferir usar fones de ouvido para conversar ao celular, parecem falar sozinhas enquanto circulam por lugares públicos.

Este artigo tem o objetivo relatar três das oito atividades criadas por uma professora brasileira com o telefone celular como ferramenta de ensino de língua inglesa entre 2009 e 2012. Estas experiências foram realizadas com adolescentes de 12 a 14 anos de idade, em uma escola de idiomas na região metropolitana do Rio de Janeiro. Neste relato serão descritas: as atividades criadas, o processo de planejamento e implementação, as dificuldades ao longo da implementação, e a reação dos estudantes às tarefas propostas. Também será descrita uma das novas tendências em ensino de idiomas: o Mobile Assisted Language Learning ou MALL, bem como possibilidades metodológicas para integração de tecnologias da informação à sala de aula. Finalmente, este artigo também abordará os ganhos pedagógicos e as dificuldades para a implementação.

\section{A história do sistema de comunicação celular}

Quem diria que uma simples parceria ao piano inspiraria uma das invenções mais transformadoras e influentes do dia-a-dia de milhares de pessoas ao redor do mundo? E quem imaginaria que esta invenção não partiria de um aclamado físico, ou engenheiro, mas sim de uma belíssima atriz de Hollywood? Ao narrar esta história, Rhodes [2011] revela como o dueto ao piano da atriz norte-americana Hedy Lamaar ${ }^{1}$ e do músico e compositor George Antheil ${ }^{2}$, em que os dois se divertiam ao tocar, simultaneamente, as mesmas notas em tons diferentes, inspirou a noção de que dois indivíduos, ao trocar informações, poderiam trocar a frequência dos sinais emitidos e ainda assim manter a

\footnotetext{
${ }^{1}$ Atriz norte-americana que atuou em diversos filmes de Hollywood entre 1930 e 1958, reconhecida como a inventora de um sistema de comunicação que serviu de base para a comunicação celular [Rhodes 2011].

${ }^{2}$ Compositor, escritor, inventor e pianista norte-americano, nascido em 1900.
} 
comunicação. Para isso, bastaria que os dois indivíduos trocassem a frequência ao mesmo tempo. Esta teoria, que constitui a base para a comunicação sem fio, foi pensada por Hedy e George com objetivos militares, já que mensagens transmitidas dessa maneira jamais poderiam ser interceptadas. Alguns anos mais tarde, em 1947, o laboratório Bell, nos EUA desenvolveu um sistema telefônico de alta capacidade interligado por diversas antenas, chamadas de células. Por isso o nome "celular".

O primeiro celular foi desenvolvido pela Ericsson em 1956, e se chamava Ericsson MTA (Mobilie Telephony A). Ele pesava cerca de 40 quilos e foi desenvolvido para ser instalado em porta malas de carros. O modelo de celular como conhecemos hoje foi desenvolvido pela norte-americana Motorola, que no dia 03 de abril de 1973, em Nova York, apresentou o modelo Motorola Dynatac 8000X [AGAR 2003].

Em 1989, existiam 4 milhões de assinantes do serviço móvel em todo o mundo. Em 2009 eram 4,6 bilhões, e estima-se que em 2013 serão 6 bilhões. A revolução propiciada pelo celular iniciou também o seu processo de digitalização [CASTELLS 1996, p. 28-69].

\section{Mobile Learning}

A mobilidade e a convergência de mídias vêm ressignificando outras modalidades, como o e-learning e o t-learning e, mais recentemente, o m-learning. De acordo Silva e Teles [2011, p. 543], o m-learning é uma evolução do e-learning e possui o mesmo objetivo, ou seja, "o de proporcionar o conhecimento em qualquer lugar e a qualquer hora; no entanto, se fundamenta na mobilidade dos dispositivos na qual a informação pode ser transmitida". O relatório Horizon [2012] aponta o m-learning, ou mobile learning, como uma tendência em educação para os próximos anos. Mobile Learning refere-se ao uso de dispositivos móveis como celulares, tablets e iPods como ferramentas de ensino e aprendizagem.

Em Tecnologia da Informação, o termo mobilidade refere-se à disponibilização de aplicações para o usuário em movimento, facultando ao mesmo a liberdade de escolher momento e local de utilização. De acordo com Geraldine Torrisi-Steele [2009, p. 3041], define-se mobile learning como a utilização de dispositivos móveis para a promoção de uma aprendizagem ativa através da geração de espaços de aprendizagem estendendo-se além das limitações físicas e temporais da sala de aula tradicional [MORAIS et al. 2011].

\subsection{Mobile Assisted Language Learning (MALL)}

$\mathrm{O}$ ensino de línguas vem sendo mediado por diferentes tecnologias que, de diferentes maneiras, subsidiam de melhor forma as quatro habilidades linguísticas: compreensão oral, fala, leitura e escrita. Nesse sentido, atualmente, é possível verificar o crescente uso de dispositivos móveis (por exemplo, celulares e tablets) para o ensino de línguas, revelando uma nova área de estudo chamada de aprendizagem de línguas mediada por dispositivos móveis, em inglês MALL, que permite maior liberdade de acesso aos usuários, que é bem retratada com a frase "em qualquer lugar e a qualquer hora" [SÁ 
et. al 2011].

No entanto, o MALL se difere do aprendizado de idiomas assistido por computador, pois se utiliza de dispositivos pessoais e portáteis, que possibilitam novos estilos de aprendizagem, enfatizando continuidade ou espontaneidade de acesso e interação em diferentes contextos [KUKULSKA-HULME and SHIELD 2008, p.4] .

Além da educação à distância, os modelos presenciais de educação também têm utilizado as tecnologias e novos formatos de conteúdo para disponibilizar materiais didáticos e criar novas experiências de aprendizagem. Esta mistura de estilos caracteriza o modelo de aprendizagem híbrida - blended learning [SQUIRRA and FEDOCE 2011, p.3].

\section{Relato das experiências}

As experiências descritas aqui tiveram início em agosto de 2009, quando uma das autoras decidiu pensar em maneiras de incorporar o telefone celular de seus alunos às próprias aulas, transformando estes aparelhos em ferramentas de ensino e aprendizagem. Este projeto foi desenvolvido nas salas de aula de uma escola de idiomas na região metropolitana do Rio de Janeiro. As atividades foram implementadas em turmas de níveis básico e intermediário, com adolescentes de 12 a 14 anos de idade. O número médio de alunos por turma participante deste projeto é de 13, e a escola, neste bairro, tem atualmente cerca de 1.200 alunos e 20 professores. Alguns destes alunos já se conheciam fora do contexto desta escola de idiomas.

Esta escola utiliza materiais didáticos desenvolvidos por sua própria editora. Os livros de onde se originaram estas lições foram Action 6 e Connexion 1, ambos da editora Learning Factory. Nesta escola o professor não interfere na elaboração do currículo ou das provas, e não escolhe o material didático que será utilizado, porém ele tem liberdade para planejar suas aulas, alterando e customizando lições, de acordo com seus objetivos. Esta escola tem as tecnologias da informação e comunicação como um dos pilares de sua missão, e quer ser uma referência no uso das TICs para ensino de língua inglesa. Todas as salas de aula estão equipadas com quadros interativos. No entanto, nesta escola, a maior parte das redes sociais, sites de jogos e softwares para chats são bloqueados. Além disso, todas as salas de aula têm cartazes proibindo o uso de celulares para conversas.

\section{Descrição das Atividades}

Estas atividades foram desenvolvidas entre os meses de agosto de 2009 e julho de 2012. Neste período foram realizados, a cada semestre, repetidamente:

- O mapeamento dos aparelhos de cada turma, bem como de suas características;

- O planejamento antecipado de todas as lições do semestre para verificação do potencial de cada lição para uso do celular com fins pedagógicos. 
No início de cada semestre foram distribuídos formulários para que os alunos sinalizassem se possuíam celulares e de que recursos dispunham em seus aparelhos. $\mathrm{O}$ preenchimento deste formulário foi feito em sala de aula, anonimamente. Como a câmera foi o recurso mais presente entre os aprendizes, e o que não representava custo de nenhum tipo para os mesmos, foram criadas as atividades descritas nos quadros 1,2 e 3 , a seguir.

\section{Quadro 01 - Atividade 01: Personalizando o material didático}

\begin{tabular}{|c|c|c|c|c|}
\hline Versão & Objetivos & Versão Original & $\begin{array}{l}\text { Versão com } 0 \\
\text { celular }\end{array}$ & Desdobramentos \\
\hline 1 & $\begin{array}{l}\text { especulação de imagens } \\
\text { através de verbos } \\
\text { modais (can, must, may } \\
\text { e might) }\end{array}$ & $\begin{array}{l}\text { Alunos especulam sobre } \\
\text { uma foto fornecida no } \\
\text { material, onde um casal } \\
\text { e uma criança posavam } \\
\text { em frente a um grande } \\
\text { prédio antigo }\end{array}$ & $\begin{array}{l}\text { Fotografarem uns aos } \\
\text { outros em locais secretos- } \\
\text { as fotos teriam que ser } \\
\text { tiradas em ângulos em que } \\
\text { eles tivessem que, em sala, } \\
\text { tentar acertar sobre em que } \\
\text { lugar do pátio selus amigos } \\
\text { estariam. }\end{array}$ & $\begin{array}{l}\text { as fotos foram transmitidas } \\
\text { para o celular da } \\
\text { professora, e depois para } \\
\text { o computador da sala de } \\
\text { aula. Dois pares não } \\
\text { possuím bluetooth, e } \\
\text { visualizaram as imagens } \\
\text { na tela do celular. }\end{array}$ \\
\hline 2 & Idem versão 1 & Idem versão 1 & \begin{tabular}{|l|} 
os alunos fotografaram \\
objetos em posiçóes \\
inusitadas e partes de \\
objetos, e, em sala de aula, \\
tiveram que especular \\
sobre quais seriam estes \\
objetos.
\end{tabular} & Idem versão 1 \\
\hline 3 & $\begin{array}{l}\text { descrição de } \\
\text { modificações feitas ao } \\
\text { corpo humano como } \\
\text { tatuagens, por exemplo, } \\
\text { utilizando a forma } \\
\text { causativa (causative } \\
\text { form) como verbo } \\
\text { HAVE. }\end{array}$ & \begin{tabular}{|l|} 
o material fornecia 7 \\
fotos de indivíduos \\
ilustrando estas \\
modificaçôes no corpo.
\end{tabular} & \begin{tabular}{|l|} 
Alunos fotografam seus \\
amigos da escola, em busca \\
de exemplos de \\
modificaçōes no corpo. \\
Lembrete da tarefa feito \\
por mensagem de texto, em \\
inglês.
\end{tabular} & $\begin{array}{l}\text { Foto tirada por alunos foi } \\
\text { exibida no quadro } \\
\text { interativo e incorporada à } \\
\text { lição. }\end{array}$ \\
\hline 4 & $\begin{array}{l}\text { Estudo de segunda } \\
\text { condicional e "I wish" ao } \\
\text { responder a pergunta: } 0 \\
\text { que você faria se } \\
\text { pudessemudar } 0 \\
\text { mundo? }\end{array}$ & $\begin{array}{l}0 \text { material fornecia } 5 \\
\text { fotos que ilustravam os } \\
\text { principais problemas do } \\
\text { mundo }\end{array}$ & $\begin{array}{l}\text { Alunos fotografaram selu } \\
\text { bairro em busca daquilo } \\
\text { que eles considerassem } \\
\text { problemas graves. }\end{array}$ & $\begin{array}{l}\text { Fotos foram exibidas no } \\
\text { quadro interativo e } \\
\text { incorporadas à lição. }\end{array}$ \\
\hline
\end{tabular}

Quadro 02 - Atividade 02: Criando um repositório de memórias 


\begin{tabular}{|c|c|c|c|c|}
\hline Versão & Objetivos & $\begin{array}{l}\text { Versão } \\
\text { Original }\end{array}$ & $\begin{array}{l}\text { Versão com o } \\
\text { celular }\end{array}$ & Desdobramentos \\
\hline Única & $\begin{array}{l}\text { Uso de } \\
\text { expressões } \\
\text { em festas. }\end{array}$ & $\begin{array}{l}\text { Conteúdo } \\
\text { exposto } \\
\text { através de } \\
\text { diálogos no } \\
\text { livro } \\
\text { didático. }\end{array}$ & $\begin{array}{l}\text { Estudantes } \\
\text { organizaram } \\
\text { uma pequena } \\
\text { festa, e } \\
\text { usaram os } \\
\text { celulares } \\
\text { para } \\
\text { fotografar } \\
\text { seus colegas } \\
\text { durante o } \\
\text { evento. }\end{array}$ & $\begin{array}{l}\text { Na aula } \\
\text { seguinte estas } \\
\text { imagens foram } \\
\text { usadas, como } \\
\text { atividade de } \\
\text { aquecimento, } \\
\text { para descrição } \\
\text { oral em } \\
\text { pares. Os } \\
\text { alunos que não } \\
\text { estavam } \\
\text { presentes } \\
\text { tiveram acesso } \\
\text { às imagens e } \\
\text { relatos sobre o } \\
\text { evento. }\end{array}$ \\
\hline
\end{tabular}

Quadro 03 - Atividade 03: Transportando Arquivos

\begin{tabular}{|c|c|c|c|c|}
\hline Versão & Objetivos & $\begin{array}{l}\text { Versão } \\
\text { Original }\end{array}$ & $\begin{array}{l}\text { Versão com o } \\
\text { celular }\end{array}$ & Desdobramentos \\
\hline Única & $\begin{array}{l}\text { A narrativa de } \\
\text { uma viagem, } \\
\text { contando } \\
\text { fatos } \\
\text { inusitados ou } \\
\text { engraçados } \\
\text { desta viagem. }\end{array}$ & $\begin{array}{l}\text { Tema } \\
\text { exposto } \\
\text { através de } \\
\text { imagens de } \\
\text { cartões } \\
\text { postais e um } \\
\text { diálogo no } \\
\text { livro } \\
\text { didático. }\end{array}$ & $\begin{array}{l}\text { Os } \\
\quad \text { aprendizes } \\
\text { foram } \\
\text { estimulados a } \\
\text { levar fotos } \\
\text { de suas } \\
\text { viagens e falar } \\
\text { sobre elas. }\end{array}$ & $\begin{array}{l}\text { As fotos } \\
\text { foram exibidas } \\
\text { no quadro } \\
\text { interativo e } \\
\text { incorporadas à } \\
\text { lição. Alunos } \\
\text { demonstraram } \\
\text { grande } \\
\text { interesse nas } \\
\text { narrativas de } \\
\text { seus colegas. }\end{array}$ \\
\hline
\end{tabular}

\section{Resultados e Discussão}

Os resultados foram avaliados de acordo com a metodologia proposta por Sprague and Dede [1999] que defendem o modelo construtivista como o modo mais efetivo para integrar tecnologia à educação. Os autores afirmam que o uso eficiente de novas tecnologias implicará na apropriação de inúmeros princípios da abordagem construtivista relacionados no Quadro 4.

\section{Quadro 04 - Resultados 01: Abordagem construtivista}




\begin{tabular}{|c|c|c|c|c|c|c|c|c|}
\hline & $\begin{array}{l}\text { Aluno } \\
\text { formula } \\
\text { regras a } \\
\text { partir da } \\
\text { observação } \\
\text { da lingua em } \\
\text { seu contexto }\end{array}$ & $\begin{array}{l}\text { Atividades } \\
\text { autênticas }\end{array}$ & $\begin{array}{l}\text { Atividades } \\
\text { centradas na } \\
\text { resolução de } \\
\text { problemas }\end{array}$ & $\begin{array}{l}\text { Integração } \\
\text { natural da } \\
\text { tecnologia à } \\
\text { aula }\end{array}$ & $\begin{array}{l}\text { Respostas e } \\
\text { conheciment } \\
\text { os dos alunos } \\
\text { conduzem } \\
\text { aula }\end{array}$ & $\begin{array}{l}\text { Alunos } \\
\text { questionam } \\
\text { uns aos } \\
\text { outros e ao } \\
\text { professor }\end{array}$ & $\begin{array}{l}\text { Pensamentos } \\
\text {, opiniões e } \\
\text { análises dos } \\
\text { alunos são } \\
\text { incorporados } \\
\text { à aula }\end{array}$ & $\begin{array}{l}\text { Engajamento } \\
\text {, autoria e } \\
\text { colaboração }\end{array}$ \\
\hline Atividade 01 & Sim & Sim & Sim & Sim & Sim & Sim & Sim & Sim \\
\hline $\begin{array}{l}\text { Atividade } 01 \\
\text { original (sem } \\
\text { os celulares) }\end{array}$ & Sim & Não & Sim & Não & Não & Não & Não & Parcialmente \\
\hline Atividade 02 & Não & Sim & Sim & Sim & Sim & $\operatorname{Sim}$ & Sim & Sim \\
\hline $\begin{array}{l}\text { Atividade } 02 \\
\text { original (sem } \\
\text { os celulares) }\end{array}$ & Não & Não & Não & Não & Não & Não & Não & Não \\
\hline Atividade 03 & Não & Sim & Não & Sim & Sim & Sim & Sim & Sim \\
\hline $\begin{array}{l}\text { Atividade } 03 \\
\text { original (sem } \\
\text { os celulares) }\end{array}$ & Não & Não & Não & Não & Parcialmente & Parcialmente & Parcialmente & Parcialmente \\
\hline
\end{tabular}

A Atividade 01 contém a maior parte dos princípios construtivistas descritos por Sprague and Dede [1999]. No entanto, foi observado que todas as atividades adquiriram contornos construtivistas, pois, em relação às atividades originais, o celular possibilitou maior integração dos questionamentos dos alunos à aula, e sua participação foi mais valorizada.

A Atividade 02 não existia na versão original - o celular proporcionou a reprodução, em sala de aula, de uma situação comum no dia a dia dos aprendizes: a lembrança de eventos passados através das fotos armazenadas no telefone. Neste caso, a mediação foi facilitada pelo visor do aparelho. Esta é uma mudança de comportamento verificada em nossa sociedade a partir do advento dos telemóveis, e assim foi reproduzida na aula de língua estrangeira. Apesar disso, esta etapa da aula destinava-se ao aquecimento e preparação dos alunos (minutos iniciais) e não, à apresentação ou prática de conteúdo.

A Atividade 03, com os celulares, proporcionou mudança real do foco das imagens pré-estabelecidas no livro para imagens levadas pelos alunos. Suas histórias e sua voz tornaram-se o centro da atividade. As imagens levadas pelo telefone proporcionaram aumento considerável no interesse pelo tema "viagens".

O Quadro 5 foi elaborado a partir de uma entrevista conduzida com os aprendizes de turmas diferentes ao final do semestre letivo. Todos (sem exceções) relataram ter feito as tarefas de casa sem dificuldades e consideraram o conteúdo fácil de compreender e as atividades mais prazerosas e divertidas quando os celulares foram utilizados. Eles também disseram que gostariam de fazer atividades deste tipo novamente. No entanto, a 
percepção dos alunos em relação às dúvidas não foi a mesma entre alunos que não utilizavam os celulares. Foi observada uma maior quantidade de dúvidas pela professora após a aula e durante a correção das tarefas de casa na aula seguinte às aulas sobre a forma causativa e sobre a descrição dos uniformes (Quadro 01). Apesar disso, o mesmo não foi observado em relação às aulas sobre verbos modais. Com ou sem celulares, os alunos apresentaram o mesmo rendimento em relação ao conteúdo. Já as atividades 02 e 03 não tiveram impacto sobre a compreensão do conteúdo, pois ambas são atividades para aquecimento (warmers) e contextualização.

\section{Quadro 05 - Resultados 02: Efetividade e aceitação dos aprendizes}

\begin{tabular}{|l|l|l|l|l|l|l|}
\hline & $\begin{array}{l}\text { Atividade } \\
\text { considerada } \\
\text { prazerosa }\end{array}$ & $\begin{array}{l}\text { Facilitou a } \\
\text { compreensão } \\
\text { do conteúdo }\end{array}$ & $\begin{array}{l}\text { Dúvidas com } \\
\text { relação ao } \\
\text { conteúdo } \\
\text { exposto }\end{array}$ & $\begin{array}{l}\text { Duração } \\
\text { influenciou } \\
\text { andamento } \\
\text { da aula }\end{array}$ & $\begin{array}{l}\text { Colaboração } \\
\text { entre alunos } \\
\text { em relação às } \\
\text { estruturas } \\
\text { introduzidas } \\
\text { no dia da } \\
\text { lição }\end{array}$ & $\begin{array}{l}\text { Proporcionou } \\
\text { vínculo entre } \\
\text { aprendizes }\end{array}$ \\
\hline Atividade 01 & Sim & Sim & Não & Sim & Sim & Sim \\
\hline $\begin{array}{l}\text { Atividade 01 } \\
\text { original (sem } \\
\text { os celulares) }\end{array}$ & Sim & Sim & Sim & Não & Sim & Não \\
\hline Atividade 02 & Sim & $\begin{array}{l}\text { Não } \\
\text { interferiu }\end{array}$ & $\begin{array}{l}\text { Não } \\
\text { interferiu }\end{array}$ & Não & Sim & Sim \\
\hline $\begin{array}{l}\text { Atividade 02 } \\
\text { original (sem } \\
\text { os celulares) }\end{array}$ & Sim & $\begin{array}{l}\text { Não } \\
\text { interferiu }\end{array}$ & $\begin{array}{l}\text { Não } \\
\text { interferiu }\end{array}$ & Não & Não & Não \\
\hline Atividade 03 & Sim & $\begin{array}{l}\text { Não } \\
\text { interferiu }\end{array}$ & $\begin{array}{l}\text { Não } \\
\text { interferiu }\end{array}$ & Sim & Não & Sim \\
\hline $\begin{array}{l}\text { Atividade 03 } \\
\text { original (sem } \\
\text { os celulares) }\end{array}$ & Sim & $\begin{array}{l}\text { Não } \\
\text { interferiu }\end{array}$ & $\begin{array}{l}\text { Não } \\
\text { interferiu }\end{array}$ & Não & Não & Não \\
\hline
\end{tabular}

A análise dos resultados parece indicar que a proposta de ensino-aprendizagem de Sprague e Dede [1999] está adequada ao uso das TICs em sala de aula. A atividade 01 foi a que apresentou uma maior quantidade de princípios construtivistas, e foi também a que mais contribuiu para a aprendizagem. Todavia, o principal ganho pedagógico entre essas atividades está no vínculo que elas proporcionam entre os alunos.

\section{Considerações Finais}

O uso de celulares, bem como o de qualquer artefato tecnológico utilizado como ferramenta de ensino e aprendizagem pressupõe: bom planejamento e capacidade de antecipação de problemas, mapeamento dos recursos disponíveis entre os aprendizes e capacidade de improvisação. O uso das TICs em sala de aula pode ser personalizado, e quanto mais for a sua adequação ao contexto, mais imprevisíveis serão os resultados. $\mathrm{O}$ 
professor que quiser se envolver com este tipo de projeto, deverá, antes de tudo, considerar o imprevisível, pois surgirão: histórias interessantes, risadas (ou até mesmo lágrimas) e lembranças. Planejar a duração de cada tarefa é fundamental, pois quanto maior a personalização, maior o tempo gasto.

Ao desenvolver projetos com TICs é fundamental que alunos e professores estejam familiarizados com a ferramenta escolhida. Quanto mais familiarizados os alunos estiverem com o recurso que o professor deseja usar (por exemplo, o vídeo ou o celular), mais fácil será a implementação do projeto. É importante incluir no planejamento tempo suficiente para instrução dos alunos com estas ferramentas e ter em mente que os alunos poderão ser grandes aliados à todas as etapas do projeto.

\section{Trabalhos Futuros}

No segundo semestre de 2010, com o avanço e popularização dos smartphones e tablets, bem como da internet $3 \mathrm{G}$, novas atividades tiveram que ser adaptadas ao contexto da sala de aula, pois devido à presença maciça de tais dispositivos, o editor de texto passou a ser utilizado. Com isso, a partir de um novo mapeamento, identificaram-se, no material didático, lições com potencial para implementação de novas atividades. Estudos recentes realizados por Jarvis [2012, p. 12] indicam que o termo Mobile Assisted Language Use (MALU) talvez seja mais apropriado que MALL, por refletir melhor a forma como os aprendizes usam a tecnologia móvel. A internet $3 \mathrm{G}$, o editor de textos, os arquivos de música, o gravador de voz e mensagens de texto constituem-se em recursos de grande potencial a serem incorporados ao ambiente de ensino de idiomas. A título de trabalhos futuros, já em desenvolvimento, apresentaremos os resultados dos projetos realizados com os novos aplicativos e analisaremos como os dispositivos móveis afetam a língua e seu uso, bem como, as suas possibilidades e desafios.

\section{Referências Bibliográficas}

Agar, J. [2003] Constant touch: a Global History of Mobile Phones. Icon Books, Cambridge, England.

Anatel, Relatório de Acompanhamento 2011. Disponível em: http://migre.me/aV2Ju. Acesso em: 19 de agosto de 2012.

Castells, M. [1996] The Rise of the Network Society. The Information Age: Economy, Society and Culture Volume I. Wiley-Blackwell. Oxford, UK.

Fedoce, R. S., e Squirra, S. C. [2011]. As tecnologias móveis e os potenciais da comunicação na educação, In: LOGOS 35. Mediações sonoras. Vol.18, No 02, pp. 267-278.

Horizon [2012] NMC Horizon Report . Acessado pela última vez em 10/08/2012.

Disponível em: http://net.educause.edu/ir/library/pdf/HR2012.pdf

Jarvis, H. [2012] Computers and Learner Autonomy: trends and issues. ELT Research Paper 12-02. British Council. London, UK.

Kukulska-Hulme, A. and Shield, L. [2008] An overview of mobile assisted language learning: From content delivery to supported collaboration and interaction.ReCALL, 20(3), pp. 271-289 [tradução das autoras]. 
Morais, D. C. S., Alencar A. D. P. C. e Souza, R. [2011] Jogo baseado em m-learning e aprendizado tangencial para auxílio ao ensino de Teoria da Computação. In: Anais do XXII SBIE - XVII WIE. Aracaju. pp. 554- 557.NMC Horizon Report 2012. Disponível em: http://net.educause.edu/ir/library/pdf/HR2012.pdf. Acesso em: $10 \mathrm{de}$ agosto de 2012.

Rhodes, R. [2011] Hedy's Folly - The Life and Breakthrough Inventions of Hedy Lamarr, the Most Beautiful Woman in the World. Illustrated, p.261. Doubleday, New York, United States.

Sá, H. F. M., Pereira, E. N. e Carelli, I. M. [2011] Aprendizagem de Inglês Assistida por Celular . In: Estudos Linguísticos e Literários: Saberes e Expressões Globais, Foz do Iguaçu, pp. 1-9.

Silva, M. e Teles, V. D. [2011] O aprendizado de língua inglesa em dispositivos móveis através da aplicação de um sistema tutor inteligente. In: Anais do XXII SBIE -XVII WIE. Aracaju, pp. 542- 545.

Sprague, D. and Dede, C. [1999] If I teach this way, am I doing my job? In: Learning and Leading with Technology. Volume 27 \#01. ISTE, pp. 6-17. 